\title{
Revision Total Knee Arthroplasty: Infection should be Ruled Out in All Cases
}

\author{
Mohammad R. Rasouli \\ Rothman Institute of Orthopaedics at Thomas Jefferson University Hospital \\ Armin Aalami Harandi \\ Rothman Institute of Orthopaedics at Thomas Jefferson University Hospital \\ Bahar Adeli \\ Rothman Institute of Orthopaedics at Thomas Jefferson University Hospital \\ James J. Purtill \\ Rothman Institute of Orthopaedics at Thomas Jefferson University Hospital \\ Javad Parvizi \\ Rothman Institute of Orthopaedics at Thomas Jefferson University Hospital \\ Follow this and additional works at: https://jdc.jefferson.edu/rothman_institute \\ Part of the Orthopedics Commons \\ Let us know how access to this document benefits you
}

\section{Recommended Citation}

Rasouli, Mohammad R.; Harandi, Armin Aalami; Adeli, Bahar; Purtill, James J.; and Parvizi, Javad, "Revision Total Knee Arthroplasty: Infection should be Ruled Out in All Cases" (2012). Rothman Institute Faculty Papers. Paper 7.

https://jdc.jefferson.edu/rothman_institute/7

This Article is brought to you for free and open access by the Jefferson Digital Commons. The Jefferson Digital Commons is a service of Thomas Jefferson University's Center for Teaching and Learning (CTL). The Commons is a showcase for Jefferson books and journals, peer-reviewed scholarly publications, unique historical collections from the University archives, and teaching tools. The Jefferson Digital Commons allows researchers and interested readers anywhere in the world to learn about and keep up to date with Jefferson scholarship. This article has been accepted for inclusion in Rothman Institute Faculty Papers by an authorized administrator of the Jefferson Digital Commons. For more information, please contact: JeffersonDigitalCommons@jefferson.edu. 


\section{Abstract:}

5 We hypothesized that some aseptic revision knee arthroplasty (TKA) failures are indeed due to

6 occult infection. This prospective study recruited 65 patients undergoing revision TKA. Mean

7 follow-up period was 19 months. Collected synovial fluid was analyzed by Ibis T5000 biosensor

8 (a multiplex PCR technology). Cases were considered as infected or aseptic based on the

9 surgeon's judgment and Ibis findings. Based on Ibis biosensor, 17 aseptic cases were indeed

10 infected that had been missed. Of these 17 cases, 2 developed infection following the index

11 revision. A considerable number of so called aseptic failures seem to be occult infections who

12 were not adequately investigated and/or miss-categorized as aseptic failure. We recommend that

13 all patients undergoing revision arthroplasty need to be investigated for PJI.

14

15 Key words: Revision knee arthroplasty, Aseptic failure, Periprosthetic joint infection, Ibis 16 T5000 biosensor, Diagnosis 


\section{Introduction}

Periprosthetic joint infection (PJI), that occurs following 1 to $3 \%$ of TKAs $[1,2]$ is the most common cause of failure after total knee arthroplasty (TKA) [3-5]. Diagnosis of PJI

21 continues to pose a challenge to the medical community because of lack of a "gold standard" [6].

22 It is, however, critical that aseptic cases be distinguished from PJI, as treatment for these 23 conditions is vastly different $[7,8]$.

History taking, physical examination, and radiographic findings can be similar in PJI and aseptic loosening and may not allow distinction in most cases [7]. Joint aspiration and serologic tests such as erythrocyte sedimentation rate (ESR) and C-reactive protein (CRP) are usually

27 performed during the work-up of a patient with suspected PJI. Although a very high sensitivity 28 has been reported for ESR and CRP, these laboratory tests have their own limitations in the 29 diagnosis of PJI [9]. Even intraoperative culture and pathology have limited sensitivity in 30 diagnosis of PJI particularly in those receiving antibiotics $[10,11]$. It has been demonstrated that 31 intraoperative culture yields false negative results in $6.6 \%$ [12] to $17.7 \%$ [13] and false positive 32 in $13 \%$ of cases [14]. Imaging modalities such as nuclear scans (tc-99, indium 111, gallium) have 33 also been employed in diagnosis of PJI but these tests are also unable to accurately differentiate 34 between aseptic loosening and septic failure after joint arthroplasty [15-17].

One of the main reasons for inability to isolate the pathogen relates to the presence of a 36 biofilm [8, 18] and/or internalization of organism by osteoblasts [19]. Biofilm is a complex 37 structure comprising microorganisms enveloped in macromolecules of glycocalyx and other 38 protective films $[18,20]$. As a result, it is probable that some of PJIs to be missed and treated as 39 aseptic failure which subsequently cause recurrent failure [21]. 
Using molecular techniques may improve diagnosis of PJI as these methods have high sensitivity and are culture independent [11]. Polymerase chain reaction (PCR) has been used in several studies to diagnose PJI [22-25]. Using a specific PCR or a broad-range (16S ribosomal DNA) PCR which are respectively able to detect only a single microorganism or previously unknown organisms were limitations of these studies. Compared to the specific PCR, the sensitivity and specificity of the broad-range PCR is lower, needs subsequent sequencing for bacterial identification, and fails to detect mixed infections [11]. Recently, the Ibis T5000 universal biosensor has been introduced as a sensitive and specific method for identification of bacteria, viruses, fungi, and protozoa. The system operates based on broad-range PCR and highperformance mass spectrometry and seems to be more accurate than conventional PCR. [26] However, it has not yet been approved by the Food and Drug Administration (FDA) for routine use in clinical practice.

The hypothesis of this study was that some cases of aseptic failure were indeed due to infection that had either escaped diagnosis using conventional modalities or had not been adequately investigated. This prospective study was set up to examine the postoperative course of a consecutive cohort of patients undergoing revision TKA in whom an intra-articular tissue and/or fluid sample was also sent for analysis by Ibis T5000 biosensor.

\section{Materials and Methods}

After approval of the Institutional Review Board of the Thomas Jefferson University, all patients who underwent TKA revision from February 2009 to May 2010 were recruited for this study. The study consists of 65 patients of whom 33 were men. The mean age of the patients was 
$6265 \pm 11$ years. All patients underwent appropriate preoperative work-up based on the

63 recommendation of the treating surgeon and then categorized as infected or uninfected based on

64 these investigations and surgeon's judgment. In our center, patients who are suspicious for PJI

65 are evaluated by measurement of serum erythrocyte sedimentation rate (ESR) and C-reactive

66 protein (CRP), intraopeartive culture and synovial fluid analysis. However, frozen section isn't

67 use as a part of PJI work-up in our institute. Intraoperatively tissue and/or fluid samples were

68 collected and analyzed using the Ibis T5000 biosensor. After discharging from the hospital,

69 patients are followed-up based on the protocol which is used routinely in our institution at 6

70 weeks, 6 months and 2 years after the revision surgery. In this study, all patients were followed-

71 up for a minimum of six months with a mean follow-up of 19 months (range; 12 to 26). In

72 particular, "aseptic" patients in whom the Ibis T5000 biosensor had detected an infecting

73 pathogen were followed-up closely for development of subsequent failure after the index

74 revision.

75 Preoperative work-up

Detailed history taking, examination, and routine radiographs were performed in all

77 patients in this cohort. It is institutional policy that all patients undergoing revision arthroplasty

78 at our institution have preoperative Erythrocyte Sedimentation Rate (ESR) and C-Reactive

79 Protein (CRP) measured. In addition, and based on the findings of serology, majority of patients

$80(\mathrm{n}=57)$ underwent joint aspiration with the fluid sent for analysis for neutrophil count, neutrophil

81 differential, and culture. As this was an observational and not interventional study, we did not

82 make any changes to the preoperative work-up of any patients. Collection of synovial fluid

83 and/or tissue sample for analysis by Ibis T5000 biosensor was a requirement of the study. 
Joint fluid and/or tissue were collected intraoperatively. Joint fluid was aspirated prior to

86

87 the arthrotomy and was also sent for WBC count (if indicated) and culture. Tissue-sampling was performed from areas that were considered by the surgeon that to be most suggestive of infection. In addition to the Ibis analysis, tissues were sent for histopathologic assessment and culture. The samples were not sent for measurement of inflammatory markers for this study. Samples then processed appropriately for later analysis by Ibis T5000 in batches. Appropriate cyrogenic vials were used to store fluid samples in a Styrofoam container. The vials were transferred in ice bags from the operating room to liquid nitrogen. About 0.5 to $1 \mathrm{~mL}$ of the liquid was stored for further analysis in liquid nitrogen. The syringe was changed in order to minimize possibility of accidental microbial contaminations before synovial fluid was transferred to the labeled vials. These vials were transferred to $-140 \mathrm{o} F$ freezer where they were stored until they were shipped in batch to the Center for Research and Genomic Studies in Allegheny, PA.

For DNA extraction, $1 \mathrm{ml}$ of the aspirate was centrifuged at 10,000 rpms for $3 \mathrm{~min}$ and 900uL of supernatant was removed. Then, ATL lysis buffer and proteinase K were added and the samples were incubated at $56^{\circ} \mathrm{C}$ until lysis occurred. The Qiagen DNeasy Tissue kit (Qiagen. Inc. cat \# 69506) was used to extract nucleic acid from the lysed sample. After DNA was extracted, $10 \mathrm{uL}$ of sample was loaded per well into each of 16 wells on the BAC detection PCR plate that each contained a different primer pair (Abbott Molecular. cat \# PN 05N13-01). The BAC detection plate is a 96 well, 6 sample plate which contains 16 primers that identify all bacterial organisms, Candida species, and determines the presence of several key antibiotic resistance markers such as van-A and van-B (vancomycin resistance) in Enterococcus species, KPC (carbapenem resistance) in Gram-negative bacteria, and mec-A (methicillin resistance) in 
107 Staphylococcus species. Once PCR was completed, the plate was loaded onto the Ibis T5000 108 machine. The products from the PCRs were desalted in a 96-well plate format and sequentially 109 electrosprayed into the time-of-flight mass spectrometer. The resultant spectral signals were then 110 processed to determine the masses of each of the PCR products present with sufficient accuracy 111 that the base composition of each amplicon could be unambiguously deduced. Using combined 112 base compositions from multiple PCRs, the identities of the pathogens and their relative 113 concentrations in the starting sample were determined.

114 The isolated microorganism from the Ibis biosensor was considered as an "orthopedic pathogen" 115 based on extensive search of the available literature. In other words, if there was any evidence 116 even a case report that shows the isolated microorganism is able to cause bone and/or joint 117 infection, that microorganism was defined as an "orthopedic pathogen". However, Ibis biosensor 118 results did not change the treatment strategy and all patients were treated based on results 119 obtained from conventional diagnostic tests and surgeon's judgment.

\section{Results}

Based on preoperative investigations and surgeon's judgment, of the 65 patients recruited

122 for this study, 21 patients were undergoing revision arthroplasty for PJI and the remaining 44 123 patients had aseptic failure. Among the 21 patients with PJI, synovial culture was negative in 11 124 cases. In the remaining 10 patients the isolated organisms were coagulase negative 125 Staphylococcus (5 cases), Staphylococcus aureus (3 patients), Streptococcus mitis plus 126 Streptococcus sanguis (1 case) and Peptostreptococcus species (1 case). Ibis identified a 127 pathogen with confidence $\geq 0.7$ in total of 36 cases. Ibis T5000 isolated an organism in 19 PJI 128 cases and failed to isolate any organism in 2 cases that were categorized as infected. The isolated 129 organism by Ibis was coagulase negative Staphylococcus in 10 patients and Staphylococcus 
130 aureus in 4 patients. In the PJI group, comparison of the isolated organisms from the culture and

131 the detected organism by the Ibis T5000 biosensor showed the same pathogens in 9 cases

132 samples whereas in 11 cases, the Ibis biosensor found additional pathogen. Table 1 demonstrates

133 comparison between isolated organism by culture and Ibis T5000 results in patients with PJI. On

134 the other hand, the Ibis T5000 found additional non-pathogen organisms in 3 cases in which the

135 Ibis T5000 had also detected an orthopedic pathogen.

All cultures in the aseptic group were negative whereas in 17 cases the Ibis T5000 found 137 orthopedic pathogens. In 27 patients, the biosensor failed to find any orthopedic pathogens. 138 Table 2 demonstrates more details on aseptic cases. During the follow-up period, 2 patients 139 failed and needed re-revision who were both initially revised for aseptic failure. The cause of 140 failure in these 2 patients was subsequent PJI with the same organism (Coagulase negative 141 Staphylococcus) as one isolated by the Ibis T5000. At the latest follow-up which ranged from 12 142 to 26 months after the index revision, all the remaining patients appear to be doing well with no 143 evidence of infection.

\section{Discussion}

Given the completely different management of aseptic loosening and PJI as well as the

147 importance of early diagnosis of PJI for establishment of a more effective treatment, 148 distinguishing between these two conditions needs special attention. Absence of a "gold 149 standard" for diagnosis of PJI [27] in addition to various defensive mechanisms of pathogens 150 such as biofilm production [8] make this differentiation more difficult. The infecting organism 151 that segregate in biofilm evade detection by conventional culture as the latter relies on isolation 152 of planktonic organisms. As a result it is suggested that some cases of PJI escape detection and 
153 erroneously are categorized as aseptic failures [8]. Although many factors contribute to 154 development of PJI after revision surgery, the latter point may be considered as one of the 155 contributing factor for the much higher incidence of PJI after revision arthroplasty than that after 156 primary replacement.

The Ibis T5000 universal biosensor is a promising technology that has been used to 158 identify a wide spectrum of pathogens in sepsis [28] and it may cover limitations of PCR method 159 for diagnosis of PJI. Because of reliance on mass specterometry and further "purification" of 160 DNA it is assumed that Ibis does not suffer the same extreme sensitivity as conventional PCR. 161 Further, because of pan-genomic amplification, Ibis may be able to detect infecting organisms 162 that could be missed by conventional PCR.

This prospective study was designed to examine the possibility of escaping some cases of 164 TKA failures which are assumed to be "aseptic" from conventional diagnostic tests. These cases 165 may be indeed infections. that have escaped diagnosis and have been miss-categorized as 166 "aseptic" failures. The study relied on Ibis T5000 for isolation of organism. Although we did not 167 accept Ibis as the "gold standard" for diagnosis of PJI, we were interested to know in what 168 percentage of patients with aseptic failure Ibis T5000 biosensor was able to isolate a pathogen. 169 Further, we sought to examine the correlation between conventional culture and Ibis in terms of 170 their ability for isolation of a pathogen and its resistance profile.

171 At our institution since 2006, we have utilized an algorithmic approach for work-up of 172 patients with failed arthroplasty which includes determination of ESR and CRP prior to revision 173 arthroplasty and selective aspiration of the failed joint in those with abnormal serology or high 174 index of suspicion for PJI [7]. In addition, intraoperative culture is performed for all cases 
175 undergoing revision arthroplasty. In spite of employing such a comprehensive and strict 176 approach, the present study revealed that a few PJIs cannot be detectable by using routine 177 diagnostic tools. It appears that reliance on conventional investigations is likely to miss occult 178 PJI at least in $30 \%$ of patients (13 out of 44 if Enterococcus faecalis cases were considered as 179 contamination). The 2 patients who were originally assumed to have aseptic failure, developed 180 infection shortly after the index revision by organisms that had been isolated by the Ibis T5000 181 but failed to be detected by conventional culture. The infecting organism in one case was low182 virulence, but a recognized pathogen [29, 30] namely Staphylococcus Caprae. Although these 183 patients did not receive any treatment for isolated additional pathogens from the Ibis biosensor, 184 our findings may indirectly indicate the clinical importance of isolated pathogens from the Ibis 185 biosensor. However, we are not able to make a statement about effect of treatment on outcome of 186 these patients with negative culture in whom the Ibis biosensor isolates additional pathogens. It 187 is possible that the conventional culture may have identified these organisms if supplemented 188 culture was utilized or the culture was kept for an extended period of time.

PCR has been used previously for the purpose of isolating organism in cases of suspected 190 PJI [22, 23, 31]. However, PCR methods suffer several limitations, most important of which is 191 the high incidence of false positive results [11]. The technique is so sensitive that it may amplify 192 contaminating and non-infecting organism such as those residing on the skin that may have been 193 picked up by aspirating needle [25]. Ibis T5000 is a multiplex PCR technology that was designed 194 to overcome some of the limitations of conventional PCR.

195 Compared to conventional PCR, the Ibis T5000 utilizes a pan-genomic amplifier that is 196 capable of isolating atypical bacteria and even non-bacterial pathogens such as fungi. Unlike 197 conventional PCR, Ibis does not rely on universal primers for amplification of DNA which may 
198 detect contaminating organism. Instead Ibis T5000 biosensor uses multiple pairs of species199 specific primers to amplify regions of an organism's genome. This process is followed by the 200 identification of that region's base composition using mass spectrometry, the results of which are 201 compared to a database which matches it to the closest microorganism [26]. The Ibis T5000 202 universal biosensor technology combines nucleic acid amplification to high-performance 203 electrospray ionization mass spectrometry and base-composition analysis. The system can 204 identify and quantify all known bacteria, all major groups of pathogenic fungi, and the major 205 families of pathogen viruses in humans and animals. Moreover, the system is capable of 206 detecting virulence factors and antibiotic resistance markers [26].

Despite its appeal, Ibis T5000 may still be a victim of high sensitivity. Ibis isolated a 208 "pathogen" in 17 cases $(38 \%)$ of "aseptic" cases. Of the latter 2 patients have failed so far due to 209 infection which we believe was missed during the index revision arthroplasty. It is possible that occult PJI may have been present in a few more cases that were either eradicated during index

211 revision arthroplasty, effectively with the patient undergoing a one stage exchange arthroplasty, 212 or are likely to manifest a failure with further follow-up. It is unlikely that the isolated organism 213 by Ibis in all 17 aseptic cases represent a true pathogen. Thus, this technology, despite its appeal, 214 should be reserved for patients in whom high index of suspicion or PJI exists but no organism 215 can be isolated. In other words the indication for use of Ibis, in our opinion, is for cases of 216 culture negative PJI. 218 the relatively short follow-up. As mentioned above, it is possible that with longer surveillance we 219 are likely to encounter more patients who may fail. Although plausible, the latter is unlikely to 220 alter the message of this study. The study highlights the importance of routine preoperative 
221 work-up using conventional serology for all and selected aspiration for some, in line with the

222 recent American Academy of Orthopedic Surgeons guidelines for diagnosis of PJI [6, 32]. It also

223 highlights the fact that a sophisticated technology is available for use by orthopedic surgeons to

224 isolate the infecting organism in cases of culture negative PJI. Another limitation of the study

225 relates to lack of a "standard" definition for PJI. It is possible that using a different diagnostic

226 criteria, some of the PJI cases in our cohort may have been considered as uninfected and vice

227 versa. The latter is unfortunately a limitation inherent to any studies related to topic of PJI as

228 various definitions for PJI exist and depending on the definition used the percent of infected

229 versus uninfected cases in a given cohort may change. It is hoped that orthopedic societies in 230 collaboration with other organizations may be able to address this shortfall in the future.

Despite the aforementioned limitations, the present study demonstrated that some aseptic

232 loosenings are not "truly" aseptic and are low grade PJIs that remain undiagnosed using

233 conventional modalities. Some of these cases may fail early for a subsequent infection. This may 234 explain the relatively high incidence of infection following revision arthroplasty, compared to 235 primary, and also the high rate of early failure of revision cases. It is thus recommended that all 236 patients undergoing revision for failed arthroplasty should be subjected to routine conventional 237 work-up which includes routine serology (ESR and CRP) and joint aspiration in patients with 238 abnormal serology tests and high index of suspicion for PJI. With further refinements of 239 molecular techniques such as multiplex PCR, the true nature of some of these so called "aseptic" 240 failures is likely to be revealed. 
244 1. Della Valle CJ, Zuckerman JD, Di Cesare PE. Periprosthetic sepsis. Clin Orthop Relat Res $245 \quad 2004 ; 420: 26$.

246 2. Phillips JE, Crane TP, Noy M, et al. The incidence of deep prosthetic infections in a specialist 247 orthopaedic hospital: a 15-year prospective survey. J Bone Joint Surg Br 2006; 88: 943.

248 3. Sharkey PF, Hozack WJ, Rothman RH, et al. Insall Award paper. Why are total knee 249 arthroplasties failing today? Clin Orthop Relat Res 2002; 7.

250 4. Bozic KJ, Kurtz SM, Lau E, et al. The epidemiology of revision total knee arthroplasty in the 251 United States. Clin Orthop Relat Res 2010; 468: 45.

252 5. Mortazavi SM, Molligan J, Austin MS, et al. Failure following revision total knee 253 arthroplasty: infection is the major cause. Int Orthop 2010 Oct 21. [Epub ahead of print];

254 6. Parvizi J, Della Valle CJ. AAOS Clinical Practice Guideline: diagnosis and treatment of 255 periprosthetic joint infections of the hip and knee. J Am Acad Orthop Surg 2010; 18: 771.

256 7. Parvizi J, Ghanem E, Menashe S, et al. Periprosthetic infection: what are the diagnostic 257 challenges? J Bone Joint Surg Am 2006; 88 Suppl 4: 138.

258 8. Nelson CL, McLaren AC, McLaren SG, et al. Is aseptic loosening truly aseptic? Clin Orthop 259 Relat Res 2005; 25.

260 9. Greidanus NV, Masri BA, Garbuz DS, et al. Use of erythrocyte sedimentation rate and C261 reactive protein level to diagnose infection before revision total knee arthroplasty. A prospective 262 evaluation. J Bone Joint Surg Am 2007; 89: 1409.

263 10. Bare J, MacDonald SJ, Bourne RB. Preoperative evaluations in revision total knee 264 arthroplasty. Clin Orthop Relat Res 2006; 446: 40. 
11. Achermann Y, Vogt M, Leunig M, et al. Improved diagnosis of periprosthetic joint infection by multiplex PCR of sonication fluid from removed implants. J Clin Microbiol 2010; 48: 1208. 12. Berbari EF, Marculescu C, Sia I, et al. Culture-negative prosthetic joint infection. Clin Infect Dis 2007; 45: 1113.

13. Trampuz A, Piper KE, Jacobson MJ, et al. Sonication of removed hip and knee prostheses for diagnosis of infection. N Engl J Med 2007; 357: 654.

14. Barrack RL, Harris WH. The value of aspiration of the hip joint before revision total hip arthroplasty. J Bone Joint Surg Am 1993; 75: 66.

15. Reing CM, Richin PF, Kenmore PI. Differential bone-scanning in the evaluation of a painful total joint replacement. J Bone Joint Surg Am 1979; 61: 933.

16. Weiss PE, Mall JC, Hoffer PB, et al. 99mTc-methylene diphosphonate bone imaging in the evaluation of total hip prostheses. Radiology 1979; 133: 727.

17. Gelman MI, Coleman RE, Stevens PM, et al. Radiography, radionuclide imaging, and arthrography in the evaluation of total hip and knee replacement. Radiology 1978; 128: 677.

18. Costerton JW, Montanaro L, Arciola CR. Biofilm in implant infections: its production and regulation. Int J Artif Organs 2005; 28: 1062.

19. Jevon M, Guo C, Ma B, et al. Mechanisms of internalization of Staphylococcus aureus by cultured human osteoblasts. Infect Immun 1999; 67: 2677.

20. Gristina AG, Costerton JW. Bacterial adherence and the glycocalyx and their role in musculoskeletal infection. Orthop Clin North Am 1984; 15: 517.

21. Parvizi J, Suh DH, Jafari SM, et al. Aseptic Loosening of Total Hip Arthroplasty: Infection Always Should be Ruled Out. Clin Orthop Relat Res 2011; 469: 1401. 
287 288

22. Tunney MM, Patrick S, Curran MD, et al. Detection of prosthetic hip infection at revision arthroplasty by immunofluorescence microscopy and PCR amplification of the bacterial 16S rRNA gene. J Clin Microbiol 1999; 37: 3281.

23. Mariani BD, Martin DS, Levine MJ, et al. The Coventry Award. Polymerase chain reaction detection of bacterial infection in total knee arthroplasty. Clin Orthop Relat Res 1996; 11.

24. Levine MJ, Mariani BA, Tuan RS, et al. Molecular genetic diagnosis of infected total joint arthroplasty. J Arthroplasty 1995; 10: 93.

25. Panousis K, Grigoris P, Butcher I, et al. Poor predictive value of broad-range PCR for the detection of arthroplasty infection in 92 cases. Acta Orthop 2005; 76: 341.

26. Ecker DJ, Sampath R, Massire C, et al. Ibis T5000: a universal biosensor approach for microbiology. Nat Rev Microbiol 2008; 6: 553.

27. Della Valle CJ, Bogner E, Desai P, et al. Analysis of frozen sections of intraoperative specimens obtained at the time of reoperation after hip or knee resection arthroplasty for the treatment of infection. J Bone Joint Surg Am 1999; 81: 684.

28. Andrade SS, Bispo PJ, Gales AC. Advances in the microbiological diagnosis of sepsis. Shock 2008; 30 Suppl 1: 41.

29. Arciola CR, Campoccia D, An YH, et al. Prevalence and antibiotic resistance of 15 minor staphylococcal species colonizing orthopedic implants. Int J Artif Organs 2006; 29: 395.

30. Shuttleworth R, Behme RJ, McNabb A, et al. Human isolates of Staphylococcus caprae: association with bone and joint infections. J Clin Microbiol 1997; 35: 2537.

31. Tarkin IS, Henry TJ, Fey PI, et al. PCR rapidly detects methicillin-resistant staphylococci periprosthetic infection. Clin Orthop Relat Res 2003; 89. 
309 32. Della Valle C, Parvizi J, Bauer TW, et al. Diagnosis of periprosthetic joint infections of the 310 hip and knee. J Am Acad Orthop Surg 2010; 18: 760.

311

312

313 